\title{
Interaction of Meloidogyne incognita and fungi: a potential threat to cotton crop in Punjab
}

\author{
Mehwish Naz ${ }^{1}$, Sajid Aleem Khan ${ }^{1, *}$, Nazir Javed and Bilquees Fatima ${ }^{2}$ \\ ${ }^{1}$ Department of Plant Pathology, University of Agriculture, Faisalabad, Pakistan; ${ }^{2}$ Institute of Horticultural Sciences, \\ University of Agriculture, Faisalabad, Pakistan \\ *Corresponding author’s e-mail: sajid_aleem@uaf.edu.pk
}

\begin{abstract}
Cotton is an important cash crop of Pakistan and is severely affected with wilt in cotton-growing areas of Punjab province. Research was carried out on two major pathogens of cotton Fusarium oxysporum f. sp. vasinfectum and Meloidogyne incognita and their possible role in wilt disease complex. During 2017-19, a survey of cotton growing areas of Punjab (Jhang, Multan, Vehari, Mianwali, Rahim Yar Khan, Bahawalpur, Kabir Wala, Dakota, Basti Dharik, Tibba Sultan Pur, Rajan Pur, Chak 27 (Layyah), Chak 99B (Layyah), Lodhran, Fateh Pur and Dagar Rohtass) was conducted to collect a sample on symptomology basis of yellowing of leaves, discoloured bark of lower part of the stem, blackened xylem vessel and wilting. Data were recorded on wilt disease incidence, prevalence, associated pathogens and root-knot nematodes. Prevalence of cotton wilt was recorded the maximum 85\% in areas of Vehari, Kabir Wala and Rajan Pur while the maximum incidence in both Multan and Tibba Sultan Pur was $15 \%$. Percentage association of wilting fungi was recorded from the soil, roots, stem and leaves on placing them in artificial media and soil dilution techniques were used to determine fungi in rhizosphere such as Fusarium oxysporum, Verticillium spp., Penicillium spp., Aspergillus spp., Rhizoctonia spp., Colletotrichum spp., Rhizopus spp., Mucor and Pythium. Root associated fungi were isolated by Hyphal tip method and isolated F. oxysporum, Rhizoctonia spp. and Pythium spp. From stem and leaf sample, F. oxysporum, Verticillium spp., Colletotrichum spp., Alternaria spp. and Botryodiplodia spp. were recorded. Relative density nematode was recorded from different locations and highest occurrence was observed in Bahawalpur (85\%) by using different extraction methods. Eggs masses of root knot nematodes were staining by using Phloxin B and nematodes inside root system was done by using acid fuchsin. Nematode reproduction parameters like No. of J2/roots system, J2 in 100ml of soils, eggs masses/root system, females/ roots system, galls/ root system were recorded. Cotton wilt caused by the interaction of Fusarium and Root knot nematode is threat to cotton industry in Pakistan and it should be treated to avoid economic losses.
\end{abstract}

Keywords: Root-knot nematode, wilt disease, cotton, Punjab, Pakistan.

\section{INTRODUCTION}

Cotton belonging to the family Malvaceae is an important fibre producing and cash crop (Lutfunnessa and Shamsi, 2011). Pakistan ranks at $4^{\text {th }}$ position among cotton production in the world, $3^{\text {rd }}$ largest exporter and also $10^{\text {th }}$ position in terms of yield (Shuli et al. 2018). Pakistan's cotton products provide for nearly $60 \%$ of its overseas earnings. About $2 \%$ of gross domestic product is reliant on cotton for the economy of Pakistan and about $10 \%$ of its value-added in agriculture (Bakhsh et al., 2009; Sial et al., 2014). In 2017-18, area under cultivation of cotton was 2,700 hectares that was an increase by 6.5 percent over last year's area $(2,489$ hectares) with estimated 11,496 million bales lower by 6.9 percent over the last year's production 10,671 million bales. Unfavourable weather, low water availability and pest attack at important stages of plant development were responsible for low cotton yield in Pakistan (Economic survey of Pakistan, 2017-2018). Root-knot nematode reduces the quantity and quality of yield. Meloidogyne incognita having wide host ranges of more than 2000-3000 species of plants and they are also obligate endoparasites. (Abad et al., 2003; Agrios, 2005). Meloidogyne spp. is the most damaging, destructive and causes up to a 5\% reduction in crop yield (Zareen et al., 2003). Sandy loam soil, shorter winter, long summer and hot climate are the most favourable for the development of disease (Maqbool, 1987). Nematodes feed on amino acids, lipids, proteins, DNA and sugar through feeding sites which/that are giant cell and syncytia (Taylor, 1990; Abawi and Chen, 1998).

Naz, M, S.A. Khan, N. Javed and B. Fatima. 2021. Interaction of Meloidogyne incognita and fungi: a potential threat to cotton crop in Punjab. Pak. J. Agri. Sci.58.1277-1290.

[Received 28 Jan 2020; Accepted 29 Jun 2021; Published (online) 21 Sep 2021]

(c) (1) 
Many fungal species can cause seedling diseases but the most important are Fusarium spp., Pythium spp., Botryodiplodia spp. and Rhizoctonia solani. These can attack before, at germination and before or after emergence (Agrios, 2005). Fusarium oxysporum., Rhizoctonia spp., Botryodiplodia., Verticillium spp. and Pythium are also reported to cause wilt complex with varying degrees of virulence. The development of disease depends on the complex interrelationship between host, pathogen and environmental conditions in all cultivated crops. In same ecological conditions interaction among soilborne pathogens with other species is common. The role of nematodes for disease development caused by soil-borne pathogens is very important and observed across the world at different crops. Fungal-nematode disease complex develops due to root-knot nematodes (Meloidogyne spp.) and many other endo and ectoparasitic nematodes associated with soilborne fungi. Previous studies conclude that nematode-fungus complexes have synergistic interaction mechanisms, biotic and abiotic factors and the use of potential management options have a significant role. An emerging threat due to root-knot nematode (M. incognita Kofoid and White (Chitwood) complex with $F$. oxysporum f. sp vasinfectum have been observed (Wang et al., 2012; Niu et al., 2008; Ulloa et al., 2013).

Interaction between $F$. oxysporum f. sp vasinfectum and Meloidogyne species was investigated by Atkinson in cotton in 1892 for the first time (Atkinson, 1892). Plant-parasitic nematodes play a vital role in the wilt disease complex in combination with plant pathogenic fungi and cause huge losses in yield. Wilt inducing fungus and root-knot nematodes act synergistically. This results in a greater combined loss than expected from either of these pathogens. Nematodes get their food from the tips or from very near to tips. These rootknot nematodes have an endoparasitic mode of action. While feeding, the female nematodes get entered into the roots. The root-knot nematodes after entering the roots, make knots on the roots. These knots or galls rupture and leave the ends of the vessels open. The openings formed now allow the entry of $F$. oxysporum into the roots. Once the fungus gains entry into the plant roots, it colonizes the vascular system of the susceptible plant. Fusarium oxysporum is specially adopted to colonize these vascular tissues of the cotton root (Smith, 1953). Hutmacher et al. (2003) investigated that root-knot nematodes are mostly present in sands and sandy loam soil. Very little work has been done in Pakistan on the interaction of wilt causing fungi and nematodes and there is a lack of information. This study provides information on cotton wilt status in Pakistan. The present study was conducted to assess the incidence of root-knot nematodes and different fungi isolated from different locations on the cotton in Punjab areas of Pakistan.

\section{MATERIALS AND METHODS}

Disease prevalence and incidence of cotton wilt: During 2017-19, cotton fields were surveyed in different areas of Punjab province, Pakistan. Sixteen locations were selected i.e Jhang, Multan, Vehari, Mianwali, Rahim Yar Khan,

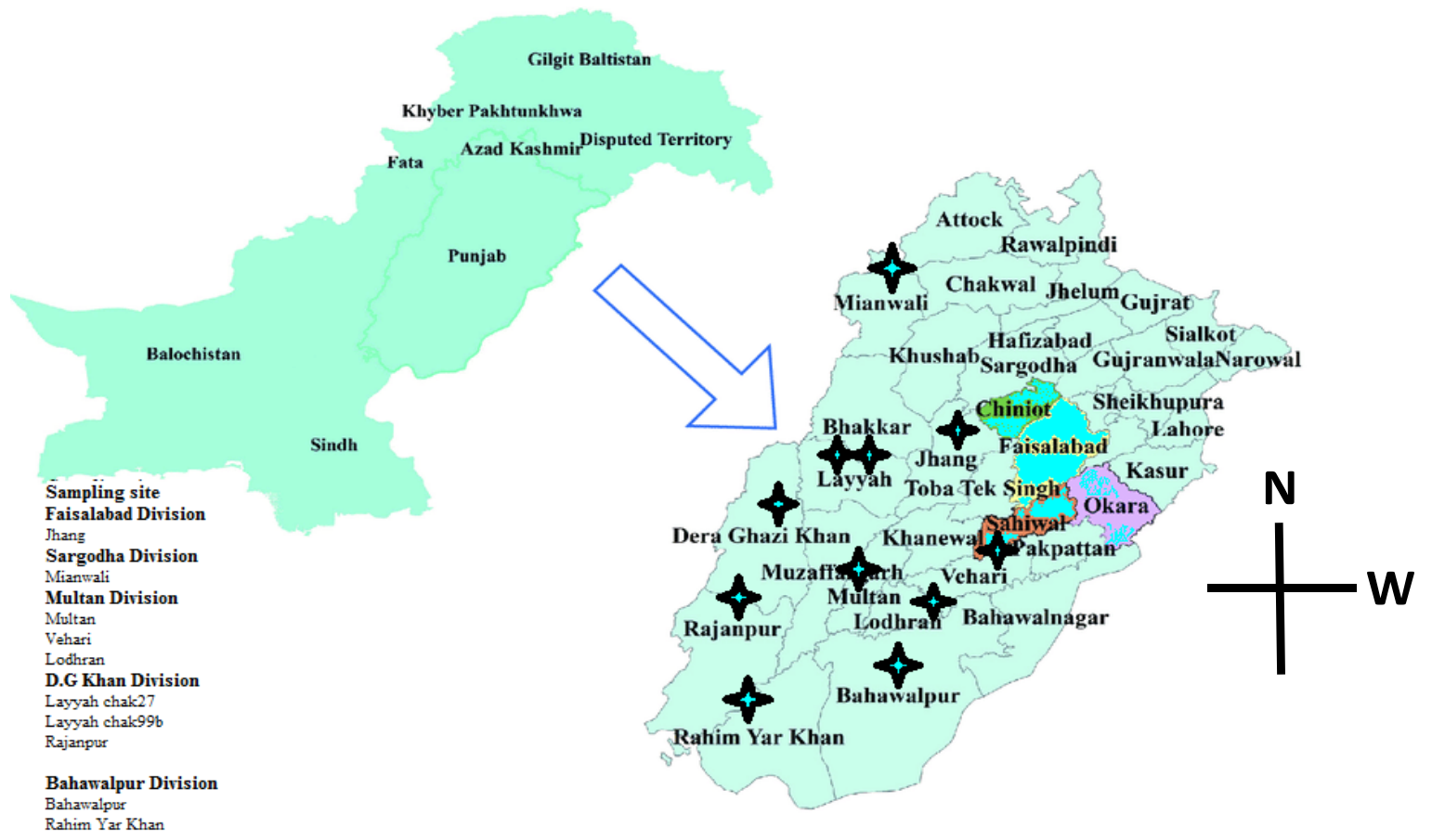

Figure 1. Indicates cotton-growing regions of Punjab surveyed during crop season 2017-2019. 
Bahawalpur, Kabir Wala, Dakota, Basti Dharik, Tibba Sultan Pur, Rajan Pur, Chak 27 (Layyah), Chak 99B (Layyah), Lodhran, Fateh Pur, Dagar Rohtass as shown in Fig.1. Cotton sample showing wilt symptoms like discolouration of vascular bundles (yellow or brown), leaf drooping, withering of plants and improper branching (Prasad and Padwick, 1939) were collected from cotton fields of Punjab, Pakistan during the crop season. From each location, five fields were selected and five samples were taken from each field randomly and made a composite sample for each field. The samples consisted of roots along with stem, leaves, and soils. The diseased samples were first packed in paper bags and then in $15 \times 20-\mathrm{cm}$ polythene bags, labelled, brought to the lab and stored at $4^{0} \mathrm{C}$ until processed for identification. Isolation, multiplication and purification of isolated pathogens were done in laboratory using artificial media and host plants.

Disease prevalence was observed to access the distribution of wilt disease in surveyed areas of Punjab regions. Following formula was used:

$$
\text { Disease Prevalence }=\frac{\text { No. of infected fields }}{\text { Fields inspected }} \times 100
$$

The disease incidence was recorded to observe the average wilt disease incidence in each field visited using the followings formula as earlier devised by Rao et al. (2016)

$$
\text { Disease incidence }=\frac{\text { No. of wilted plants }}{\text { Total plants examined }} \times 100
$$

Isolations, identification and frequency percentage of most prevalent fungi associated with cotton wilt: Plant Pathogenic fungi were isolated from infected root tissues, soils, stem and leaf of cotton. Isolation of plant pathogenic fungi isolated from soil sample (Warcup, 1955). Small pieces of diseases, as well as healthy root tissue from infected roots of cotton plants collected during the survey, was cut with the help of a sterilized scalpel. Mercuric chloride $(0.1 \%)$ were used to sterilize cut tissues of infected cotton plants for 30-60 seconds. Excessive mercuric chloride was removed through rinsing of the sample in distilled water. These pieces of infected roots were placed on Petri plates containing PDA \{(Potato dextrose agar media) (potato starch, $20 \mathrm{~g}$; agar, $20 \mathrm{~g}$; dextrose, $20 \mathrm{~g}$ and distilled water $1 \mathrm{~L})\}$ was used for isolation of plant pathogenic fungi from infected plant samples collected from various locations. Isolated fungi were purified by using the single spore technique and hyphal tip method and incubated at $28 \pm 2^{\circ} \mathrm{C}$ in an incubator. The culture was preserved for further use at PDA slants in refrigerator $4{ }^{\circ} \mathrm{C}$ (Kulkarni, 2006).

The frequency of each isolated fungus from plant part on PDA was calculated as earlier reported by Saxena and Singh (1987).

$$
\begin{aligned}
& \text { Relative frequency (\%) } \\
& =\frac{\text { No. of pieces colonized by each fungus }}{\text { Total number of pieces placed on media }} \times 100
\end{aligned}
$$

A single spore technique was used for the transfer of a single germinated conidium to obtain a pure culture. This method is suitable for species of fungal genera that produce spores in culture, for example, Fusarium, Colletotrichum, Alternaria, Stemphylium, Bipolaris, Verticillium and Phoma (Choi et al., 1999).

The hyphal tipping method was also used that involves the transfer of a single hyphal tip to obtain a pure culture. This method is suitable for species of fungal genera such as Pythium, Rhizoctonia, Sclerotium and Sclerotinia (Wosten et al., 1991).

Occurrence and frequency percentage of root-knot nematodes: Samples of soils with infected roots were collected. These composite sample of soil was preserved in $15 \times 20-\mathrm{cm}$ polythene bags. Polythene bags were airtight to maintain humidity. After proper labelling, brought to Nematology Laboratory, Department of Plant Pathology, University of Agriculture, Faisalabad. The roots were separated from the soil and washed with water for isolation of fungi and nematodes. The relative frequency of nematodes (Anwar and McKerny, 2012) was calculated on the following:

Frequency of nematode (\%)

$$
=\frac{\text { Number of samples with RKN }}{\text { Total number of samples }} \times 100
$$

Isolation and identification of Meloidogyne spp. from infected root samples: For nematode isolation from soil and root samples, Whitehead and Hemming tray method (Whitehead and Hemming, 1965) and Baermann funnel method (McKenry and Roberts, 1985) was used. Data on nematode development (Number of galls, J2s, females, egg masses per root system were recorded. Identification of nematodes was done on the basis of their morphological structures. Staining of female within root for identification perineal pattern slides was made (Hartman and Sasser, 1985). Plant roots were washed gently under running tap water to remove soil particles and prepared clearing solution of Lactophhenol (phenol 20g, lactic acid 20ml, Glycerine 40ml, distilled water $20 \mathrm{ml}$ ) to boil and add $5 \mathrm{ml} 1 \%$ solution of acid fuchsin or $0.1 \%$ cotton blue using a glass beaker on hot plate. Leave it for 3 minutes and then washed. Examined under microscope and counting of nematodes was done under a stereomicroscope (Olympus SZ 61) at 3.5X magnification and then it was multiplied by two, to get total number of nematodes / root system (Bridge et al., 1981).

Statistically analyses: Statistics 8.1 software was used to statistically analyse the data. One way ANOVA was applied and Least Significant Difference test at 5\% level of significance was used at $5 \%$.

\section{RESULTS}

Disease prevalence and incidence of cotton wilt:

Disease prevalence: The prevalence of wilt was calculated in different fields of cotton growing areas of Punjab based on symptoms. Maximum disease prevalence was recorded in Vehari, Kabir Wala, Rajan Pur and Chak 99B (Layyah) (85\%) 
and which were non significantly $(\mathrm{P}<0.05)$ different from each other. Both Dakota (D.G Khan) Tibba Sultan Pur (Vehari) showed $80 \%$ disease prevalence and which were non significantly $(\mathrm{P}<0.05)$ different from each other. Both Basti Dharik and Chak 27 (Layyah) showed 65\% wilt disease prevalence and which were non significantly $(\mathrm{P}<0.05)$ different from each other. Mianwali showed by $60 \%$ followed by Bahawalpur $(57 \%)$ disease prevalence while it was recorded $47 \%$ in Multan. Dagar Rohtass and Fateh Pur showed $45 \%$, Rahim Yar Khan $46 \%$ and Lodhran $41 \%$. The minimum disease was recorded in Jhang 36\%. All visited places showed varying disease prevalence and were significantly different from each other at $P \leq 0.05$ as shown in Fig. 2.
Disease incidence: All the places observed for disease incidence showed variability. Maximum incidence of wilt was observed $15 \%$ in Tibba Sultan followed by Multan $14.8 \%$, Vehari (13.4\%) and Bahawalpur (13.2\%). Lodhran showed a $12 \%$ incidence followed by Jhang showed (10.2\%). Chak 99B (Layyah) (9\%), Mianwali $8.4 \%$ and Rajan Pur 8.2\%. Both Dakota and Chak 27B (Layyah) showed a 7.2\% incidence and were non significant $(\mathrm{P}<0.05)$ from each other. Rahim Yar Khan showed 7\% followed by Kabir Wala 5.2\%. Both Fateh Pur and Dagar Rohtass 5.6\% and were not significant $(\mathrm{P}<0.05)$ from each other. The minimum incidence of wilt was recorded in Basti Dharik (4\%). The results were significant from each other at $P \leq 0.05$ as shown in Fig. 3 .

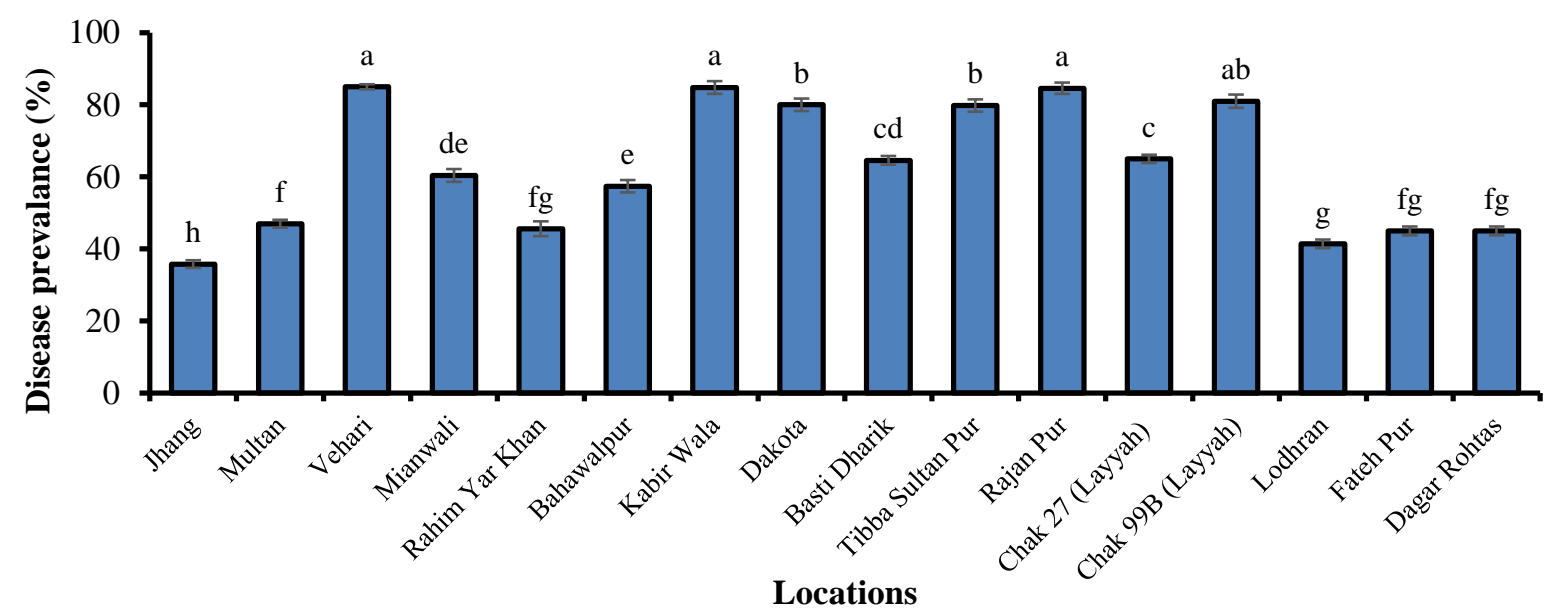

Figure 2. Percentage of disease prevalence in cotton-growing regions of Punjab during survey 2017-19. Means sharing similar letters are statistically non-significant at $(\mathrm{P}<0.05)$ according to Least Significant Difference and vertical bars give standard error (SE) of means.

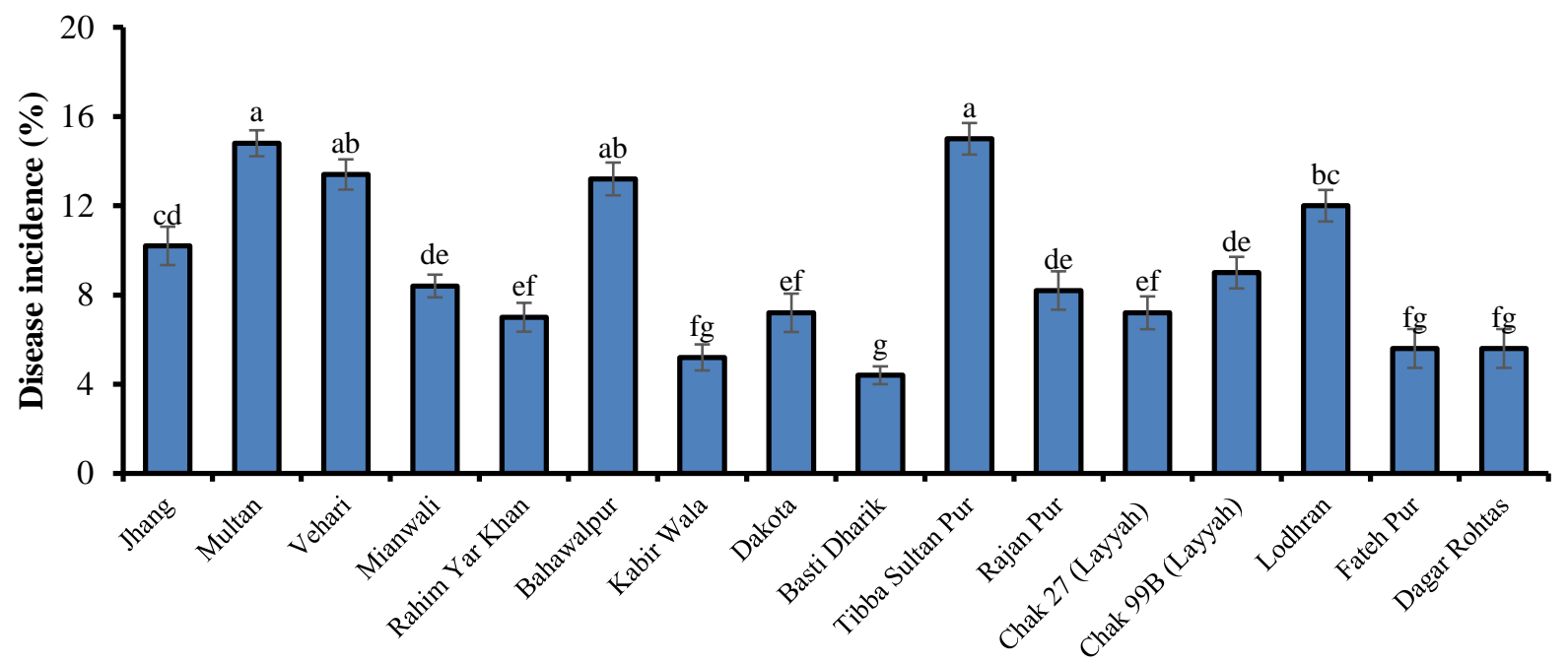

Locations

Figure 3. Percentage of disease incidence in cotton-growing regions of Punjab during survey 2017-19. Means sharing similar letters are statistically non-significant at $(\mathrm{P}<0.05)$ according to Least Significant Difference and vertical bars give standard error (SE) of means. 
Frequency \% of different most prevalent fungi associated with cotton wilt from root samples: Maximum frequently of $F$. oxysporum was dominating among isolated fungi was recorded 65.2\% in Dagar Rohtass (D.G khan) followed by Chak 99B (Layyah) 60.2\%, Chak 27 (Layyah) (58.6\%), Rajan Pur (56.8\%), Fateh Pur 58.2\% and Dakota (56.4\%). That results were significantly different from each other. Both areas of Punjab Kabir Wala and Basti Dharik was recorded $54.4 \%$ frequency of $F$. oxysporum and which were non significantly $(\mathrm{P}>0.05)$ different from each other In Bahawalpur (52.2\%), Lodhran 52\%, Multan 45.2\%, Rahim Yar Khan $44.8 \%$, Vehari $35.6 \%$ was recorded. The minimum frequency of $F$. oxysporum recorded in Jhang $30.4 \%$.

Rhizoctonia spp. were also found associated with root samples was recorded $38.6 \%$ in Rajan Pur followed by Chak 27 (Layyah) (35.6\%), Layyah Chak 99B (32\%). Both Fateh Pur and Multan showed (30.2\%) which were non significantly (P > 0.05) different from each other, Tibba Sultan Pur 27\%, Vehari 25\%, Bahawalpur 23.8\%, Dakota 22.8\%, Kabir Wala 22.2\%, Basti Dharik 20.4\%, Rahim Yar Khan 18.4\%, Dagar Rohtass $16.8 \%$. The minimum frequency of $R$. bataticola was observed $14.4 \%$ in Jhang.

Pythium 31.8\% (Rajan Pur) followed by Chak 27 (Layyah) 25\%, Layyah chak 99B 24\%, Kabir Wala 23.8\%, Dagar Rohtass 23.2\% Fateh Pur 22.2\% Bahawalpur 19.4\%,
Bahawalpur 19.4\%, Vehari 16.2\%, Multan 13.8\%, Basti Dharik 19\%, Jhang 12\%, Rahim Yar Khan $11 \%$ was recorded. Minimum Pythium frequently 7.2\% in Lodhran was recorded. The results were significantly different from each other at $P \leq$ 0.05 as shown in Fig. 4.

Frequency \% of fungi associated with cotton: Percentage of F. oxysporum was higher in Dagar Rohtass (D.G Khan) 74\% followed Dakota (66\%), Multan and Basti Dharik 65.6\%. Chak 99B (Layyah) 65.2\%, Chak 27 (Layyah) 63.2\%, Kabir Wala 62.6\%, Rajan pur $60.8 \%$, Tibba Sultan Pur 58.6\%, Bahawalpur 58.2\%, Lodhran 55.2\%, Mianwali 55\% Rahim Yar khan 51.8\%, Vehari 58.2\%. Minimum frequently isolated fungi of $F$. oxysporum was recorded $44.8 \%$ in Jhang, Frequency percentage was significantly different $(P \leq 0.05)$ at all locations.

As far as Verticillium spp. is concerned, the highest percentage association was estimated in Tibba Sultan (37\%) followed by Rajan Pur and Lodhran (33\%), Bahawalpur, Basti Dharik (32\%) while the Verticillium spp frequency in other areas including Vehari, Rahim Yar Khan, Mianwali Dakota, Jhang, Kabir Wala and Chak 99B (Layyah), Fateh Pur and Chak 27 (Layyah), Multan, Dagar Rohtass showing statistically significant $(P \leq 0.05)$ results ranging between 31 $26 \%$ respectively. On the other hand, Multan and Dagar Rohtass exhibited lower percentage association of

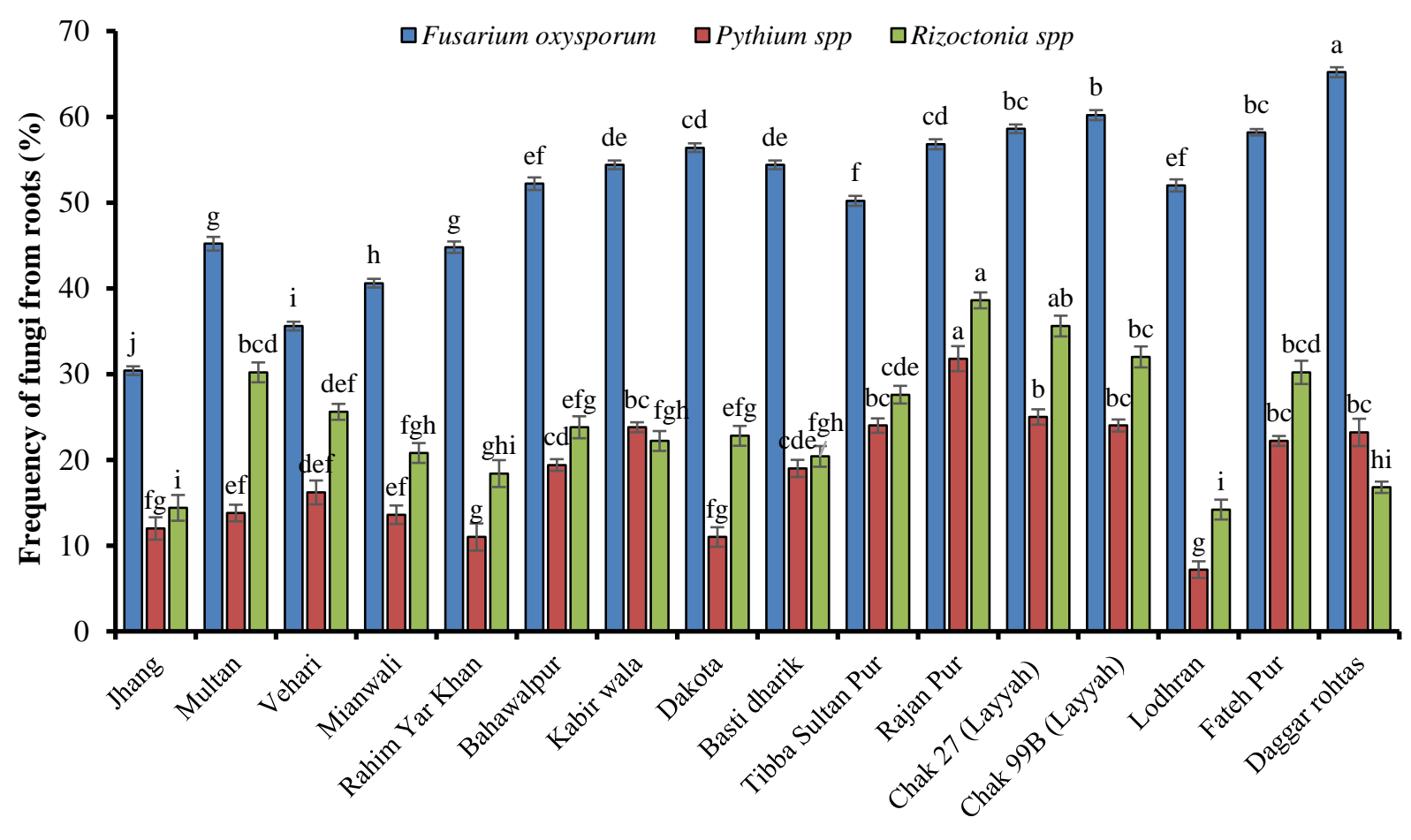

Locations

Figure 4. Frequency percentage of isolated fungi (root samples) in different locations. Means sharing simillar letters are statistically non-significant at $(\mathrm{P}<0.05)$ according to Least Significant Difference and vertical bars give standard error (SE) of means. 
Verticillium spp. Likewise, higher frequency for Penicillium spp was recorded in Jhang (16\%) followed by Multan, Rahim Yar Khan, Bahawalpur and Dakota showed (14\%), Vehari, Mianwali, Tibba Sultan Pur, Chak 27 (Layyah) and Lodhran (13\%). In addition, Kabir Wala and Chak 99B (Layyah), Fateh Pur and Dagar Rohtass showed 12\% frequency for Penicillium spp, however, significantly different $(P \leq 0.05)$ from each other. Nevertheless, samples collected from Rajan Pur showed the lower percentage (7\%) for Penicillium spp. Samples collected from different cotton growing areas of Punjab also showed Aspergillus spp frequency in Rahim Yar Khan (38.4\%) followed by Bahawalpur, Mianwali, Vehari, Kabir Wala, Tibba Sultan Pur, Basti Dharik, Chak 99B (Layyah), Lodhran, Chak 27 (Layyah) Lodhran and Dagar Rohtass ranging 37-20\%. On other hand, lowest percentages were recorded in Fateh Pur, Jhang, Dokota and Rajan Pur between 19 to $6.2 \%$ respectively, however, these results are significantly different $(P \leq 0.05)$ from each other. Nevertheless, samples collected from Rajan Pur showed the lower percentage (6.2\%) for Aspergillus spp.

As far as R. Bataticola is concerned, the highest percentage association was recorded in Tibba Sultan Pur (50\%) followed by Basti Dharik (48\%), Kabir Wala (46\%) and Multan (41\%) while the $R$. Bataticola frequency in other areas including Dakota, Jhang, Bahawalpur, Vehari, Mianwali, Rahim Yar Khan, Lodhran showing slightly lower percentages which were statistically similar results ranging between 40-23\% respectively. On the other hand, Dagar Rohtass exhibited lower percentage association $(3 \%)$ for R. Bataticola.

Colletotrichum spp. 16.6\% (Vehari), Rahim Yar Khan 16.4\% Mianwali $15.2 \%$ Jhang 15\% Multan $14.8 \%$ Bahawalpur 13.6\% Rajan Pur 13.4\% Tibba sultan 12.6\% Basti Dharik 11.6\% Chak 99B (Layyah) 11\%, Kabir Wala 10.4\%, Chak 27 (Layyah)10.2\% Dakota 9.8\% Dagar Rohtass 9.2\% Fateh Pur $8.6 \%$, Lodhran $8.4 \%$ were recorded.
Rhizopus percentage was estimated higher in Mianwali (69\%) followed by Bahawalpur, Vehari, Rahim Yar Khan, Basti Dharik, Dakota, Rajan Pur, Multan and Lodhran and Tibba Sultan Pur showing ranging 67-53\% respectively. However, the lower percentage (40\%) for Rhizopus spp. was recorded from collected samples of Chak 99B (Layyah).

Moreover, the Mucor spp. was also detected from collected samples in which samples from Mianwali represents higher percentage (27\%) followed by Jhang, Multan, Vehari, Rahim Yar Khan, Bahawalpur, Kabir Wala, Dakota, and Basti Dharaik depicting 16-20\% of pathogen. Although, the areas such as Dagar Rohtass and Rajan Pur revealed lower percentage (13\%) for Mucor spp. from collected samples.

Pythium spp. percentage was 33\% in Rajan Pur followed by Fateh Pur, Dagar Rohtass, Chak 27 (Layyah), Chak 99B (Layyah), Jhang Multan, Vehari, Mianwali, Rahim Yar Khan, Bahawalpur, Kabir Wala, Dakota, Basti Dharik, Tibba Sultan Pur, Rajan Pur (25-12\%). However, the lower percentage (9\%) for Pythium spp. was recorded from collected samples of Lodhran. The results were significantly different from each other at $P \leq 0.05$ as shown in Table 1 .

Frequency percentage of fungi associated with cotton stem and leaf samples: From stem and leaf sample, Verticillium spp, Colletotrichum spp and Alternaria spp were recorded in different areas of Punjab.

Percentage of $F$. oxysporum was higher in Dagar Rohtass (D.G Khan) $70 \%$ followed by Dakota (62\%), Basti Dharik and Fateh Pur 61.4\%. Chak 99B (Layyah) 61.2\%, both Multan and Kabir wala 60.2\%, Chak 27 (Layyah) 60\%, both Bahawalpur and Rajan pur $56.8 \%$ Frequency percentage was not significantly $(\mathrm{P}<0.05)$ different from each other. Tibba Sultan Pur 55.4\%, Mianwali 51\%, Vehari 50.8\%, Rahim yar khan $48.2 \%$, Lodhran $45.6 \%$, Minimum frequently isolated fungi of $F$. oxysporum was recorded $40.2 \%$ in Jhang,

Table.1. Frequency percentage of isolated fungi (soil sample) at different locations.

\begin{tabular}{|c|c|c|c|c|c|c|c|c|c|}
\hline Locations & $\begin{array}{c}\text { Fusarium } \\
\text { oxysporum }\end{array}$ & $\begin{array}{c}\text { Verticillium } \\
\text { spp. }\end{array}$ & $\begin{array}{c}\text { Penicillium } \\
\text { spp. }\end{array}$ & $\begin{array}{c}\text { Aspergillus } \\
\text { spp. }\end{array}$ & $\begin{array}{c}\text { Rizoctonia } \\
\text { spp. }\end{array}$ & $\begin{array}{c}\text { Colletotrich } \\
\text { um spp. }\end{array}$ & $\begin{array}{c}\text { Rhizopus } \\
\text { spp. }\end{array}$ & $\begin{array}{c}\text { Mucor } \\
\text { spp. }\end{array}$ & $\begin{array}{c}\text { Pythium } \\
\text { spp. }\end{array}$ \\
\hline Jhang & $44.8 \mathrm{j}$ & $27 \mathrm{efg}$ & $16 a$ & $17.8 \mathrm{e}$ & $36 \mathrm{~d}$ & $15.0 \mathrm{ab}$ & $43 \mathrm{i}$ & $16 \mathrm{fg}$ & $12 \mathrm{ef}$ \\
\hline Multan & $65.6 b$ & $24 \mathrm{i}$ & $14 a-d$ & $20.4 d$ & $41 \mathrm{c}$ & $14.8 \mathrm{ab}$ & $54 f$ & 20de & $14 \mathrm{e}$ \\
\hline Vehari & $51.8 \mathrm{i}$ & $31 \mathrm{~cd}$ & $13 b-e$ & $32.6 b$ & $32 \mathrm{ef}$ & $16.6 \mathrm{a}$ & $66 b$ & $26 b$ & $17 \mathrm{~cd}$ \\
\hline Mianwali & $55 \mathrm{~h}$ & $28 \mathrm{ef}$ & $13 b-e$ & $34.6 b$ & $30 f g$ & $15.2 \mathrm{ab}$ & $69 \mathrm{a}$ & $27 \mathrm{a}$ & $15 \mathrm{de}$ \\
\hline Rahim Yar Khan & $51.8 \mathrm{i}$ & 29de & $14 \mathrm{abc}$ & $38.4 \mathrm{a}$ & $28 \mathrm{~g}$ & $16.4 \mathrm{a}$ & $65 c$ & $23 \mathrm{c}$ & $12 \mathrm{ef}$ \\
\hline Bahawalpur & $58.2 \mathrm{~g}$ & $32 \mathrm{bc}$ & $14 a-d$ & $36.8 \mathrm{a}$ & $35 \mathrm{de}$ & $13.6 \mathrm{bc}$ & $67 b$ & 20de & $20 \mathrm{c}$ \\
\hline Kabir wala & 62.6de & $26 f g h$ & $12 \mathrm{e}$ & $25.6 \mathrm{c}$ & $46 b$ & $10.4 \mathrm{fgh}$ & $54 \mathrm{ef}$ & $16 f g$ & $24 \mathrm{~b}$ \\
\hline Dakota & $66 b$ & $28 \mathrm{ef}$ & $14 \mathrm{ab}$ & $2.84 \mathrm{~g}$ & $40 \mathrm{c}$ & $9.8 f-i$ & $58 \mathrm{~d}$ & 14hi & $12 \mathrm{ef}$ \\
\hline Basti Dharik & $65.6 \mathrm{~b}$ & $32 \mathrm{bc}$ & $11 \mathrm{a}$ & $24.6 \mathrm{c}$ & $48 \mathrm{ab}$ & 11.6def & $59 \mathrm{~d}$ & $20 \mathrm{e}$ & $19 \mathrm{c}$ \\
\hline Tibba Sultan Pur & $58.6 \mathrm{fg}$ & $37 \mathrm{a}$ & $13 b-e$ & $25.0 \mathrm{c}$ & $50 \mathrm{a}$ & $12.6 \mathrm{cde}$ & $53 \mathrm{f}$ & $22 \mathrm{~cd}$ & $25 b$ \\
\hline Rajan Pur & $60.8 \mathrm{ef}$ & $33 b$ & $7 f$ & $6.2 \mathrm{f}$ & $21 \mathrm{~h}$ & $13.4 \mathrm{bcd}$ & $56 \mathrm{e}$ & 13hi & $33 a$ \\
\hline Layyah Chak 27 & $63.2 \mathrm{~cd}$ & 25hi & $13 b-e$ & $20.8 \mathrm{~d}$ & $11 \mathrm{i}$ & $10.2 \mathrm{f}-\mathrm{i}$ & $44 \mathrm{i}$ & $17 f$ & $25 b$ \\
\hline Layyah Chak 99B & $65.2 \mathrm{bc}$ & $26 \mathrm{gh}$ & $12 \mathrm{cde}$ & $21.6 \mathrm{~d}$ & $12 \mathrm{i}$ & $11.0 \mathrm{efg}$ & $40 \mathrm{j}$ & $15 \mathrm{gh}$ & $24 b$ \\
\hline Lodhran & $55.2 \mathrm{~h}$ & $33 b$ & $13 b-e$ & $21.0 \mathrm{~d}$ & $23 \mathrm{~h}$ & $8.4 \mathrm{i}$ & $54 \mathrm{f}$ & $11 \mathrm{j}$ & $9 f$ \\
\hline FatehPur & $65 \mathrm{bc}$ & $25 \mathrm{ghi}$ & $12 \mathrm{de}$ & 19.0be & $12 \mathrm{i}$ & 8.6hi & $49 \mathrm{~h}$ & $23 \mathrm{c}$ & $24 b$ \\
\hline Dagar Rohtass & $74 \mathrm{a}$ & $23 \mathrm{i}$ & $12 \mathrm{e}$ & $20.2 \mathrm{~d}$ & $3 \mathrm{j}$ & $9.2 \mathrm{ghi}$ & $51 \mathrm{~g}$ & $13 \mathrm{ih}$ & $23 \mathrm{~b}$ \\
\hline
\end{tabular}

Means sharing similar letters are statistically non-significant at $(\mathrm{P}<0.05)$ according to Least Significant Difference. 
Frequency percentage was significantly $P \leq 0.05$ different at all locations.

Verticillium spp was isolated from these locations. Rajan Pur was $27.8 \%$ followed by Jhang 23.6\%, Multan 19.6\%, Vehari 26.2\%, Mianwali 27.8\%, Rahim Yar Khan 24.2\%, Bahawalpur 26.2\%, Kabir Wala 21.8\%, Dakota 25\%, Basti Dharik 27\%, Tibba Sultan 19\%, Rajan Pur 27.4\%, Chak 27 (Layyah) 15.8\%, Chak 99B (Layyah) 17.4\%, Lodhran 18.6\%, Fateh pur $21.8 \%$, Dagar Rohtass $20.2 \%$ and were results significantly from each other at $P \leq 0.05$. Colletotrichum spp in Dakota 25.6\% Kabir Wala 22\% Tibba sultan 19.6\% Vehari $18.8 \%$ Fateh Pur 18.4\% Bahawalpur 18.2\% Multan and Chak 27 (Layyah) and Lodhran 17\%, Dagar Rohtass 16.6\%, Basti Dharik 16\% Jhang 15.6\% Rajan Pur 14.8\% Chak 99B (Layyah) $13.6 \%$, Rahim yar khan $12.6 \%$ Mianwali $10.6 \%$. These were results significant from each other at $P \leq 0.05$ Alternaria spp. (35.6\%) in Basti Dharik were recorded. Chak 99B (Layyah) 33, Chak 27 (Layyah) 32, Dakota 28.8\%, Dagar Rohtass 25.4\% Rajan Pur 21.6\%, Fateh Pur 23.6 Jhang 17.2\% Bahawalpur Mianwali 16.4\% Bahawalpur 14.6\% Tibba sultan $13.6 \%$ Kabir wala $11.4 \%$ Vehari $10.8 \%$ Lodhran 10.6 Rahim yar khan $10.4 \%$ Multan $8 \%$. These were results significant from each other at $P \leq 0.05$. Maximum frequently isolated wilted fungi from roots Botryodiplodia $34.2 \%$ in Kabir Wala followed by which Fateh Pur showed 30\%, Rajan Pur 28.6\%, Bahawalpur 26\%, Mianwali 24.8\%, Vehari 22\%, Basti Dharik 18.8\%, Jhang 16\%, Dakota 15.8\%, Rahim Yar Khan 15.2\%, Layyah Chak 27 (14.6\%), Tibba Sultan Pur (13.8\%). The minimum least frequency of Botryodiplodia in Lodhran showed $12 \%$ as shown in Table 2.

Occurrence of Populations of nematodes from infected roots: Result of survey from different visited locations indicated that mean comparison of total number of non-stylet bearing/ 100ml soil recorded from visited locations were the maximum in Chak 99B (Layyah) (37) followed by Lodhran (25), Chak 27 (Layyah) (21) and Dokta (18). Kabir Wala and Multan showed (15) was non significantly $(\mathrm{P}<0.05)$ different from each other while it was recorded (13) in Basti Dharik. However minimum number of non-stylet bearing/ 100ml soil recorded in Mianwali, Vehari and Rahim Yar Khan.

Means comparison of stylet bearing and non stylet bearing nematodes were also assessed and results indicated that stylet bearing root knot nematodes $/ 100 \mathrm{ml}$ were recorded maximum in Dagar Rohtas (874) followed by Rahim Yar Khan (817), Basti Dharik (789) and Jhang (784). Multan showed 732 while it was recorded 643 in Chak 99B (Layyah). Minimum number of stylet bearing root knot nematodes/100ml were recorded in Rajan Pur and Tibba Sultan Pur. Pratylenchus $/ 100 \mathrm{ml}$ soil were recorded maximum in Kabir Wala (19) followed by Bahawalpur, Mianwali and Dakota (17) was non significantly $(\mathrm{P}<0.05)$ different from each other while minimum number in Rajan Pur. Number of Helicotylenchus spp. was the highest found in Jhang (7) followed by Multan and Basti Dharik (6) was non significantly $(\mathrm{P}<0.05)$ different from each other while minimum in Mianwali, Rajan Pur and Chak 99B (Layyah). All visited places showed were significantly different from each other at $(\mathrm{P} \leq 0.05)$ as shown in Table 3 .

Occurrence and frequency \% of $M$. incognita: The results of the survey of different locations indicated that the maximum nematode population was recorded $85 \%$ in Bahawalpur, $84.2 \%$ in Vehari, $82.6 \%$ in Multan which was non significantly $(\mathrm{P}<0.05)$ different from each other. $80.2 \%$ in Dagar Rohtass, $76.2 \%$ in Rahim Yar Khan. Chak 27 (Layyah) 75\%, Mianwali 66\% Lodhran 60\% Fateh Pur 55.4\%, Jhang $50 \%$, Tibba Sultan Pur 35.2\%, Kabir Wala 34\%. Both Basti

Table 2. Frequency percentage of fungi associated with cotton stem and leaf sample

\begin{tabular}{|c|c|c|c|c|c|}
\hline Locations & $\begin{array}{c}\text { Fusarium } \\
\text { oxysporum }\end{array}$ & Verticillium spp. & $\begin{array}{c}\text { Collectotrichum } \\
\text { spp. }\end{array}$ & Alternaria spp. & Botryodiplodia spp. \\
\hline Jhang & $40.2 \mathrm{~h}$ & $23.6 \mathrm{~cd}$ & $15.6 \mathrm{c}-\mathrm{f}$ & $17.2 \mathrm{ef}$ & 16.0hi \\
\hline Multan & $60.2 c$ & $19.6 \mathrm{efg}$ & $17.0 \mathrm{~b}-\mathrm{e}$ & $8.0 \mathrm{i}$ & $31.4 \mathrm{ab}$ \\
\hline Vehari & $50.8 \mathrm{e}$ & $26.2 \mathrm{abc}$ & $18.8 \mathrm{bcd}$ & $10.8 \mathrm{ghi}$ & $22 \mathrm{efg}$ \\
\hline Mianwali & $51.0 \mathrm{e}$ & $27.8 \mathrm{a}$ & $10.6 \mathrm{f}$ & $16.4 \mathrm{efg}$ & $24.8 \mathrm{def}$ \\
\hline Rahim Yar Khan & $48.2 \mathrm{f}$ & $24.2 \mathrm{~cd}$ & $12.6 \mathrm{ef}$ & 10.4hi & 15.2hi \\
\hline Bahawalpur & $56.8 \mathrm{~d}$ & $26.2 \mathrm{abc}$ & $18.2 \mathrm{~b}-\mathrm{e}$ & $14.6 \mathrm{fgh}$ & $26.0 \mathrm{cde}$ \\
\hline Kabir Wala & $60.2 c$ & $21.8 \mathrm{de}$ & $22.0 \mathrm{ab}$ & $11.4 \mathrm{ghi}$ & $34.2 \mathrm{a}$ \\
\hline Dakota & $62.0 \mathrm{~b}$ & $25.0 \mathrm{bc}$ & $25.6 \mathrm{a}$ & $28.8 \mathrm{bc}$ & 15.8hi \\
\hline Basti Dharik & $61.4 b c$ & $27.0 \mathrm{ab}$ & $16.0 c-f$ & $35.6 \mathrm{a}$ & $18.8 \mathrm{gh}$ \\
\hline Tibba Sultan Pur & $55.4 \mathrm{~d}$ & $19.0 \mathrm{fg}$ & $19.6 b c$ & $13.6 f-i$ & $13.8 \mathrm{i}$ \\
\hline Rajan Pur & $56.8 \mathrm{~d}$ & $27.4 \mathrm{ab}$ & $14.8 \mathrm{c}-\mathrm{f}$ & $21.6 \mathrm{de}$ & $28.6 \mathrm{bcd}$ \\
\hline Layyah Chak 27 & $60.0 \mathrm{c}$ & $15.8 \mathrm{~h}$ & $17.0 \mathrm{~b}-\mathrm{e}$ & $32.0 \mathrm{ab}$ & 14.6hi \\
\hline Layyah Chak 99B & $61.2 \mathrm{bc}$ & $17.4 \mathrm{gh}$ & 13.6def & $33.6 \mathrm{ab}$ & $20.6 f g$ \\
\hline Lodhran & $45.6 \mathrm{~g}$ & $18.6 \mathrm{fg}$ & $17.0 \mathrm{~b}-\mathrm{e}$ & 10.6hi & $12.0 \mathrm{i}$ \\
\hline Fateh Pur & $61.4 \mathrm{bc}$ & $21.8 \mathrm{de}$ & $18.4 \mathrm{bcd}$ & $23.6 \mathrm{~cd}$ & $30.0 \mathrm{abc}$ \\
\hline Dagar Rohtas & $70.0 \mathrm{a}$ & $20.2 \mathrm{ef}$ & $16.6 \mathrm{~b}-\mathrm{e}$ & $25.4 \mathrm{~cd}$ & $22.4 \mathrm{efg}$ \\
\hline
\end{tabular}

Means sharing simillar letters are statistically non-significant at $(\mathrm{P}<0.05)$ according to Least Significant Difference. 
Naz, Khan, Javed \& Fatima

Table 3. Nematode population in different cotton growing areas of Punjab

\begin{tabular}{|c|c|c|c|c|c|}
\hline \multirow[t]{2}{*}{ Locations } & \multirow{2}{*}{$\begin{array}{c}\text { Non-Stylet } \\
\text { Bearing /100 } \\
\text { mL soil }\end{array}$} & \multicolumn{4}{|c|}{ Stylet bearing } \\
\hline & & RKN/100 mL soil & $\begin{array}{c}\text { Pratylenchus } / 100 \mathrm{~mL} \\
\text { soil }\end{array}$ & $\begin{array}{l}\text { Helicotylenchus } \\
/ 100 \mathrm{~mL} \text { soil }\end{array}$ & Others/100 mL soil \\
\hline Jhang & $13 \mathrm{c}-\mathrm{f}$ & $784 b c$ & $11 \mathrm{~cd}$ & $7 \mathrm{a}$ & 19de \\
\hline Multan & $15 c-f$ & $732 c$ & $8 \mathrm{cdef}$ & $6 a b$ & $21 \mathrm{cde}$ \\
\hline Vehari & $11 \mathrm{def}$ & $639 d$ & $13 b c$ & $4 \mathrm{bcd}$ & $30 \mathrm{bc}$ \\
\hline Mianwali & $10 \mathrm{ef}$ & $646 \mathrm{~d}$ & $17 \mathrm{ab}$ & $2 \mathrm{de}$ & $15 \mathrm{e}$ \\
\hline Rahim Yar Khan & 9f & $817 \mathrm{ab}$ & 9 cde & 3 cde & $12 \mathrm{e}$ \\
\hline Bahawalpur & $11 \mathrm{def}$ & $630 \mathrm{~d}$ & $17 \mathrm{ab}$ & $5 a b c$ & $14 \mathrm{e}$ \\
\hline Kabir Wala & $15 c-f$ & $542 \mathrm{e}$ & $19 \mathrm{a}$ & $3.2 \mathrm{cde}$ & $31 \mathrm{~b}$ \\
\hline Dakota & $18 \mathrm{~b}-\mathrm{e}$ & $647 d$ & $17 \mathrm{ab}$ & $4 \mathrm{bcd}$ & $27 \mathrm{bcd}$ \\
\hline Basti Dharik & 13def & $789 b c$ & $13 b c$ & $6 a b$ & $15 \mathrm{e}$ \\
\hline Tibba Sultan Pur & $12 \mathrm{def}$ & $439 \mathrm{f}$ & $8 \mathrm{cdef}$ & 3 cde & $21 \mathrm{cde}$ \\
\hline Rajan Pur & $11 \mathrm{def}$ & $466 f$ & $5 \mathrm{ef}$ & 2de & $17 \mathrm{e}$ \\
\hline Layyah Chak 27 & $21 b c$ & $548 \mathrm{e}$ & $7 \mathrm{def}$ & $5 b c$ & $33 a b$ \\
\hline Layyah Chak 99B & $37 \mathrm{a}$ & $643 d$ & $3 \mathrm{f}$ & $1 \mathrm{e}$ & $42 \mathrm{a}$ \\
\hline Lodhran & $25 \mathrm{~b}$ & $659 \mathrm{~d}$ & $8 \mathrm{cdef}$ & $4 \mathrm{bcd}$ & $33 \mathrm{ab}$ \\
\hline FatehPur & $11 \mathrm{def}$ & $655 \mathrm{~d}$ & $11 \mathrm{~cd}$ & 3 cde & $13 \mathrm{e}$ \\
\hline Dagar Rohtas & $18 \mathrm{bcd}$ & $874 a$ & $13 b c$ & $5 a b c$ & $31 b c$ \\
\hline
\end{tabular}

Means sharing similar letters are statistically non-significant at $(\mathrm{P}<0.05)$ according to Least Significant Difference.

Dharik 26\% and Dakota $24.6 \%$ which was non significantly $(\mathrm{P}<0.05)$. However, number of nematodes in Chak 99B (Layyah) 73\%, Rajan Pur 72\% which was non significantly $(\mathrm{P}<0.05)$. All results were significant from each other at $(P \leq 0.05)$ as shown in Fig. 5.

Nematodes Reproduction parameters: Number of $\mathrm{J}_{2 s}$ of $M$. incognita from roots: Maximum $\mathrm{J}_{2 \mathrm{~s}}$ from roots were recorded in Dagar Rohtas (2528) and Rahim Yar Khan (2431) which were non significantly $(\mathrm{P}<0.05)$ different from each other. Jhang showed (2275) followed by Multan (1986), Bahawalpur (1626), Vehari (1615), Lodhran (1541) and Kabir Wala (1329). Number of $\mathrm{J}_{2 \mathrm{~s}}$ isolated from Basti Dharik (1929), Fateh Pur (1824) and Mianwali (1819) which were non significantly $(\mathrm{P}<0.05)$ different from each other. Both Rajan Pur (1453) and Layyah Chak 27 (1426) which were non significantly $(\mathrm{P}<0.05)$ different from each other. However,

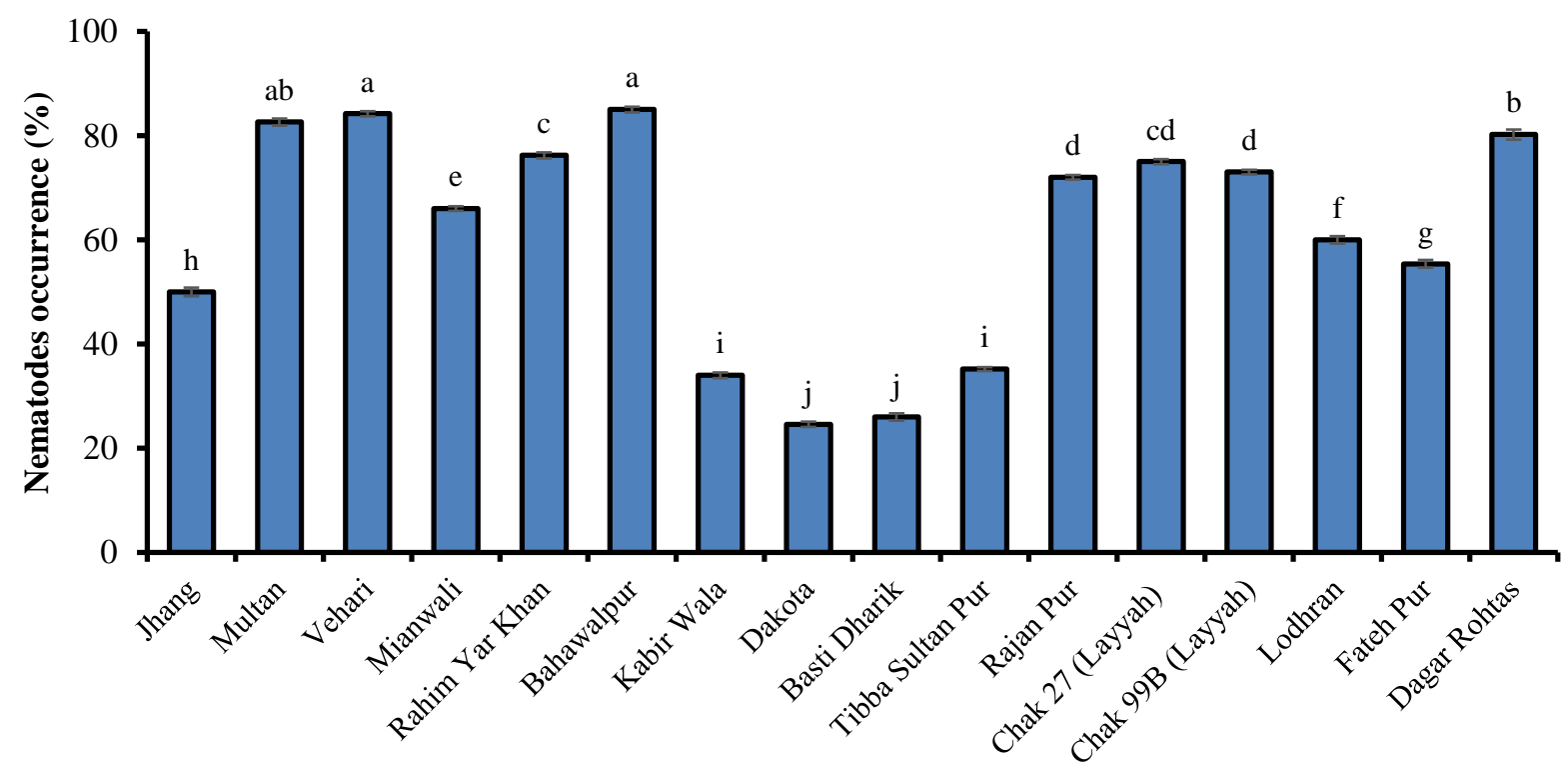

Locations

Figure 5.Percentage Occurrence of nematodes at different locations. Means sharing similar letters are statistically nonsignificant at $(\mathrm{P}<0.05)$ according to Least Significant Difference and vertical bars give standard error $(\mathrm{SE})$ of means. 
Dakota (1732), Layyah Chak 99b (1723) which were non significantly $(\mathrm{P}<0.05)$ different from each other. The minimum number of $\mathbf{J}_{2 \mathrm{~s}}$ was calculated in Tibba sultan (966). Data showed a significant difference among values at $(\mathrm{P} \leq$ 0.05) as shown in Fig. 6.

Number of $J_{2 s}$ of $M$. incognita from $100 \mathrm{ml}$ soils: Maximum no of J2s were observed in Multan (732) and next was Mianwali (646), Vehari (639), Layyah Chak 27 (548), Kabir Wala (542), Rajan Pur (466). Jhang (784), Rahim Yar Khan
(817), Basti Dharik (789) and Dagar Rohtass (874) which were non significantly $(\mathrm{P}<0.05)$ different from each other. No of $\mathrm{J}_{2 \mathrm{~s}}$ of $M$. incognita from soils Dakota (647) and Bahawalpur (630) and Layyah Chak 99b (643) which were non significantly $(\mathrm{P}<0.05)$ different from each other. Lodhran (659) and Fateh Pur (655) which were non significantly $(\mathrm{P}<0.05)$ different from each other. The minimum number of $\mathbf{J}_{2 \mathrm{~s}}$ was collected from the soil sample in Tibba Sultan Pur (439) as shown in Fig. 7.

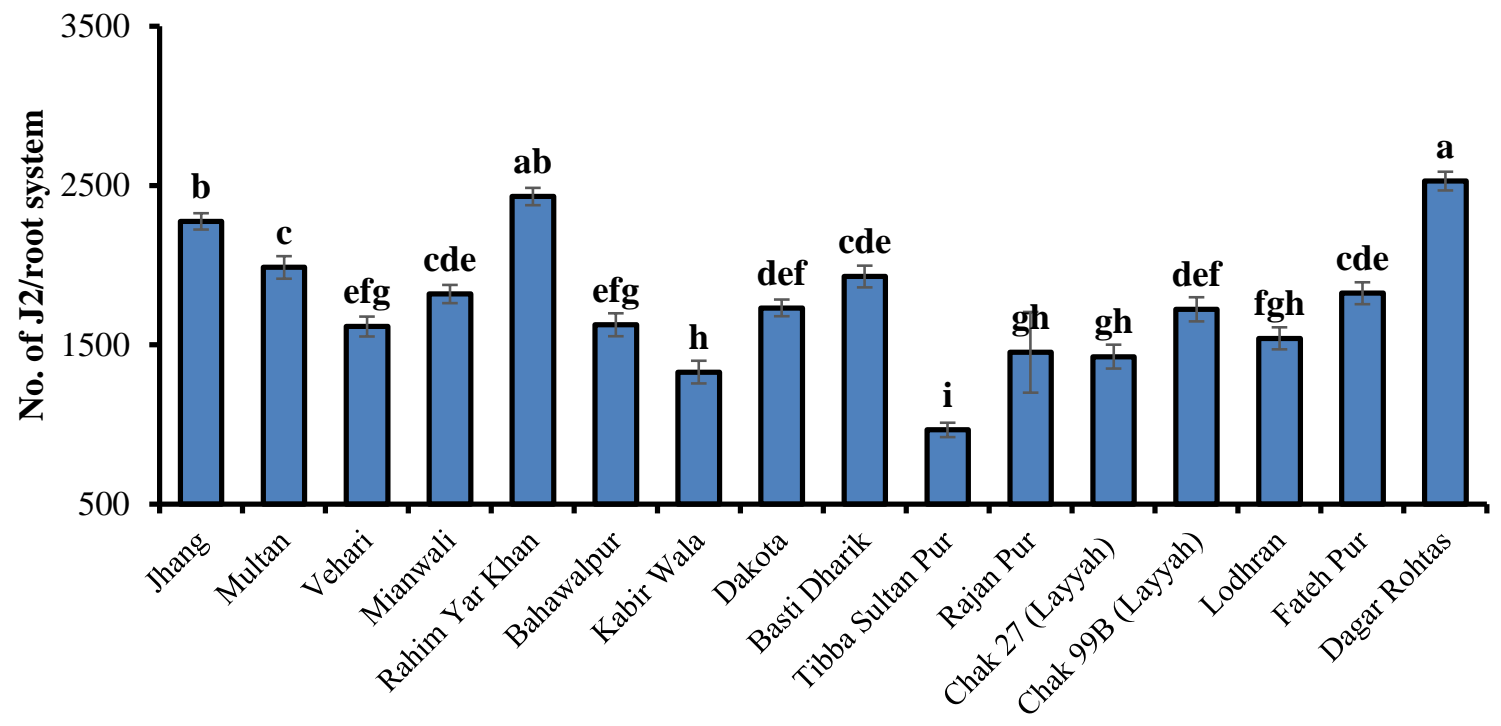

Locations

Figure 6. Number of $\mathbf{J}_{2 \text { s }}$ from roots counted in the sample from different locations. Means sharing similar letters are statistically non-significant at $(\mathrm{P}<0.05)$ according to Least Significant Difference and vertical bars give standard error $(\mathrm{SE})$ of means.

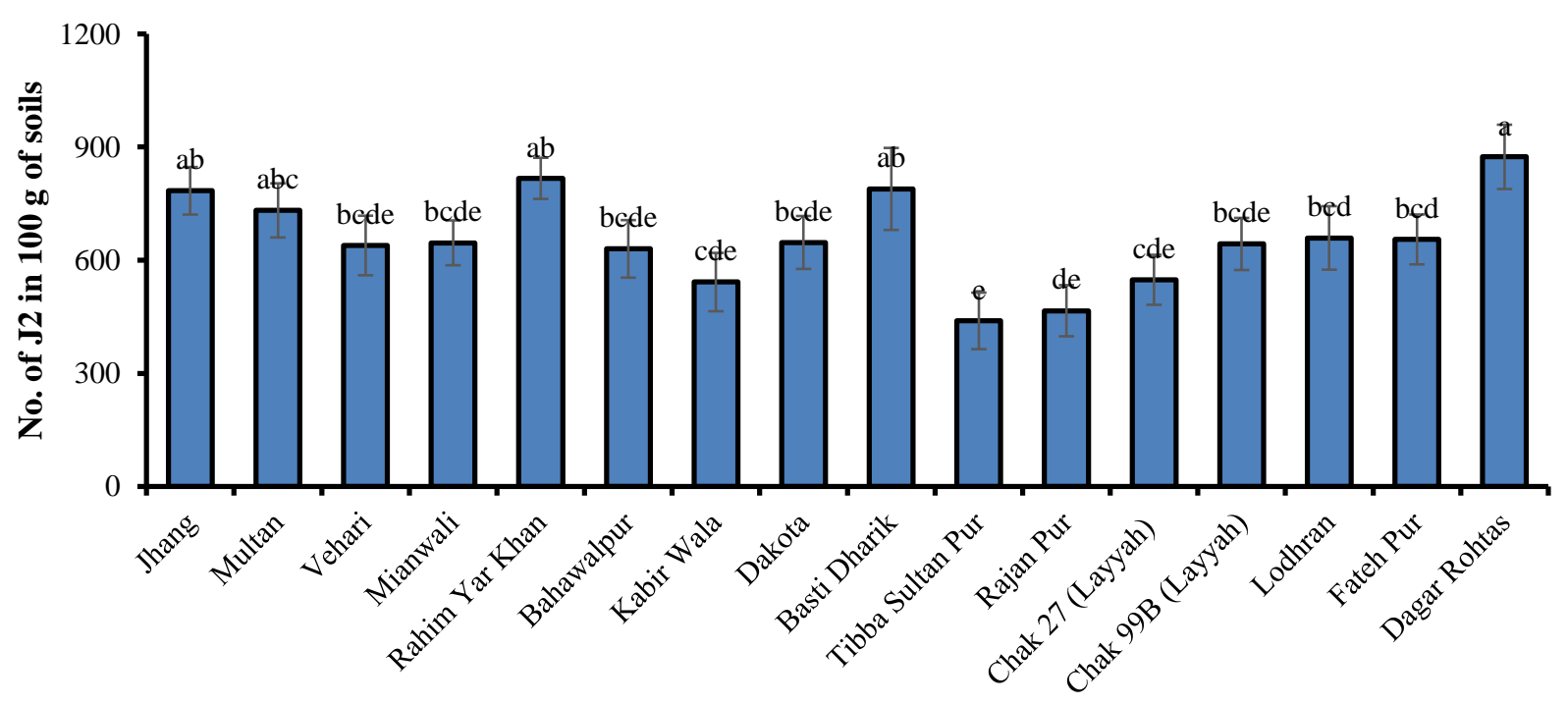

Locations

Figure 7. No of $\mathbf{J}_{2 \mathrm{~s}}$ from $100 \mathrm{ml}$ soils counted from different locations. Means sharing similar letters are statistically nonsignificant at $(\mathrm{P}<0.05)$ according to Least Significant Difference and vertical bars give standard error $(\mathrm{SE})$ of means. 
No of egg masses: The highest number of egg masses in Multan (48), Basti Dharik (43), Mianwali (42), Fateh Pur (41), Dakota (39), both Bahawalpur and Lodhran (38), Layyah Chak 99b (37), Vehari (35), Kabirwala (31). Number of egg masses were recorded in Jhang (58) followed by both Rahim yar khan and Dagar Rohtas (55) which were non significantly $(\mathrm{P}>0.05)$ different from each other. Likewise, both Layyah Chak 27 (28) and Rajan Pur (26) which were non significantly
$(\mathrm{P}<0.05)$ different from each other. Minimum number of egg masses (23) was recorded in Tibba sultan as shown in Fig. 8. No of females: The highest number of females was recorded in Rahim Yar Khan (77) and Dagar Rohtas (78) which was non significantly $(\mathrm{P}<0.05)$ different from others followed by Jhang 72, Multan (61), Basti Dharik (59), Layyah Chak 99b (54), Vehari (50), Bahawalpur (49), Lodhran (48), Vehari (35). Number of females was recorded in Kabir Wala (41) and Layyah Chak 27 (42) which was non significantly $(\mathrm{P}<0.05)$

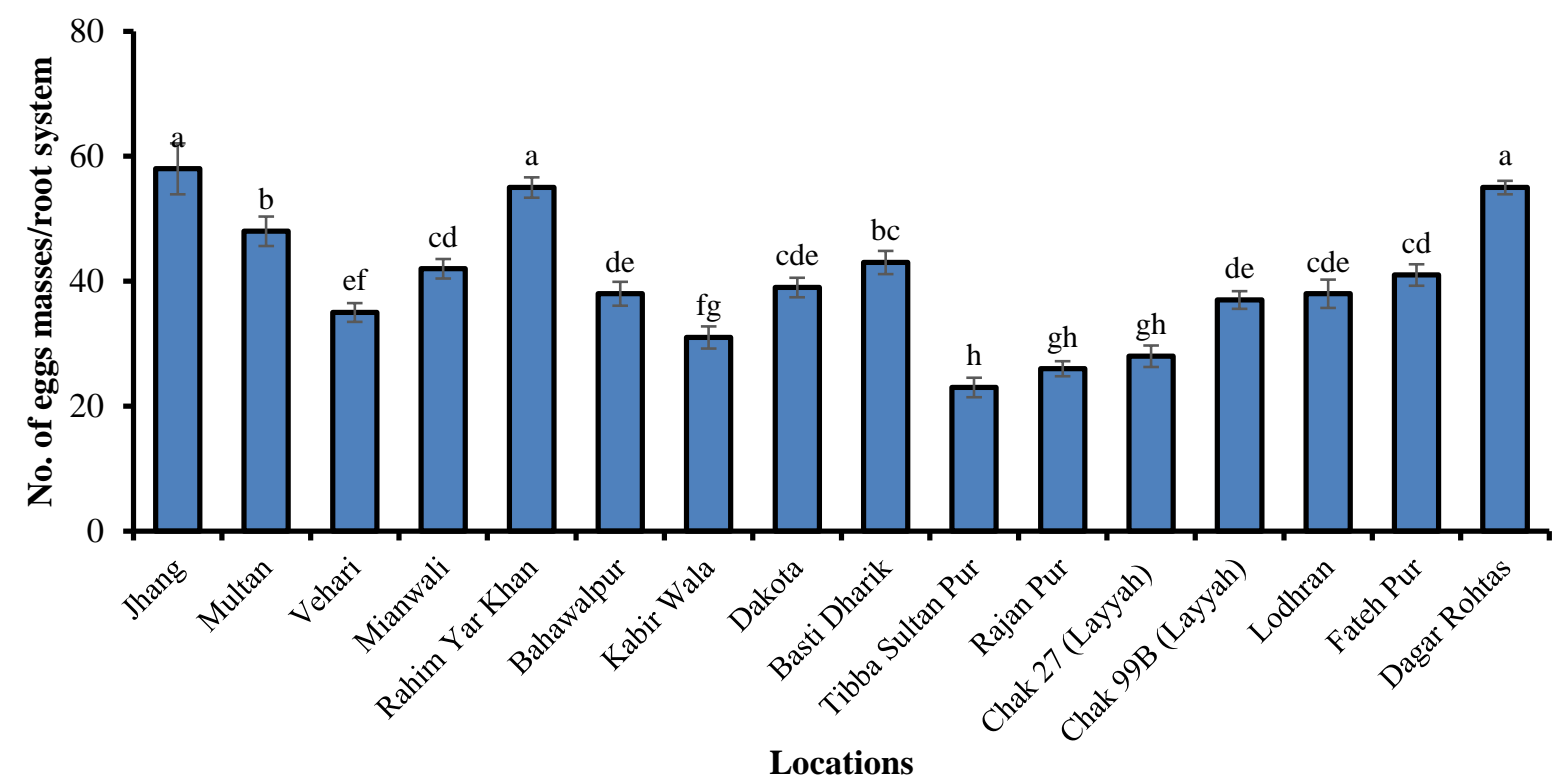

Figure 8. Number of egg masses observed in cotton root samples at different locations. Means sharing similar letters are statistically non-significant at $(\mathrm{P}<0.05)$ according to Least Significant Difference and vertical bars give standard error $(\mathrm{SE})$ of means.

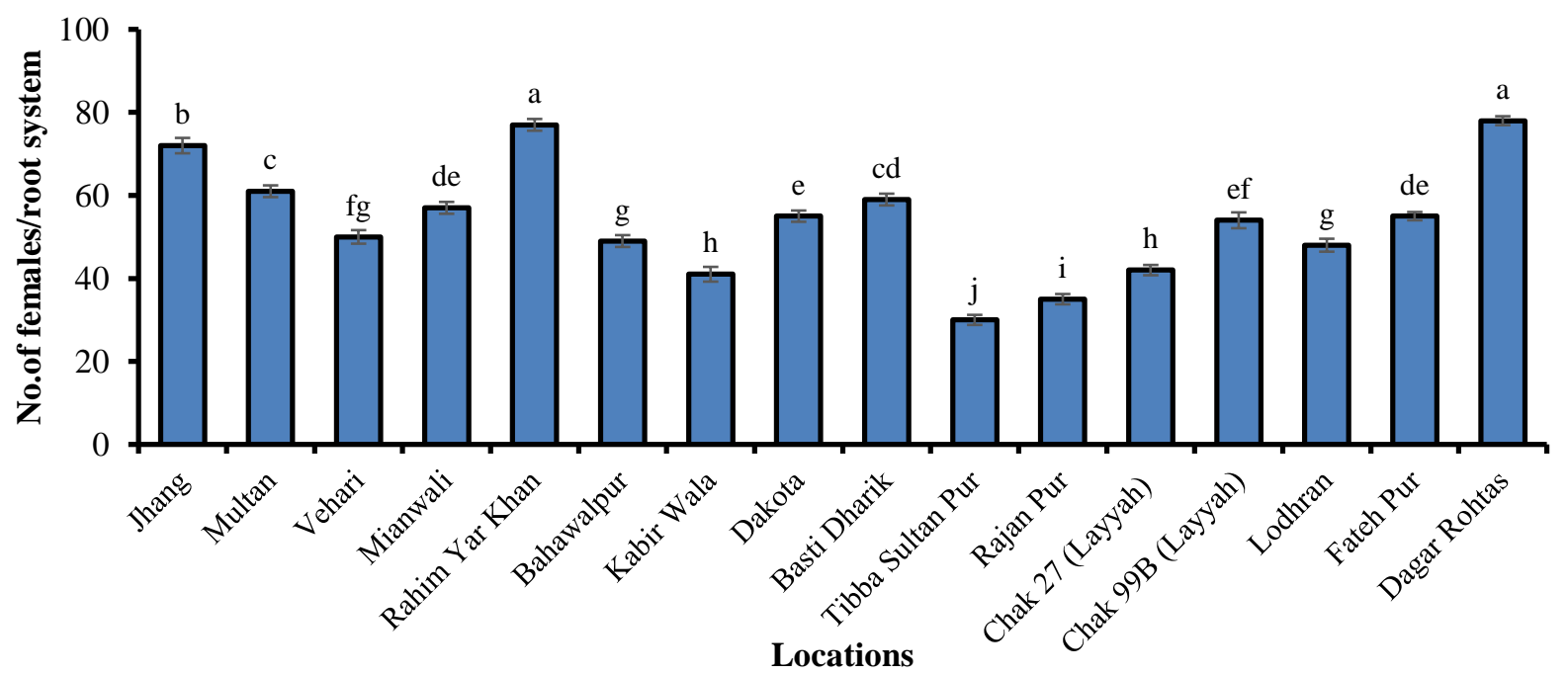

Figure 9. No of females/Root system isolated from cotton root samples at different locations. Means sharing similar letters are statistically non-significant at $(\mathrm{P}<0.05)$ according to Least Significant Difference and vertical bars give standard error (SE) of means. 


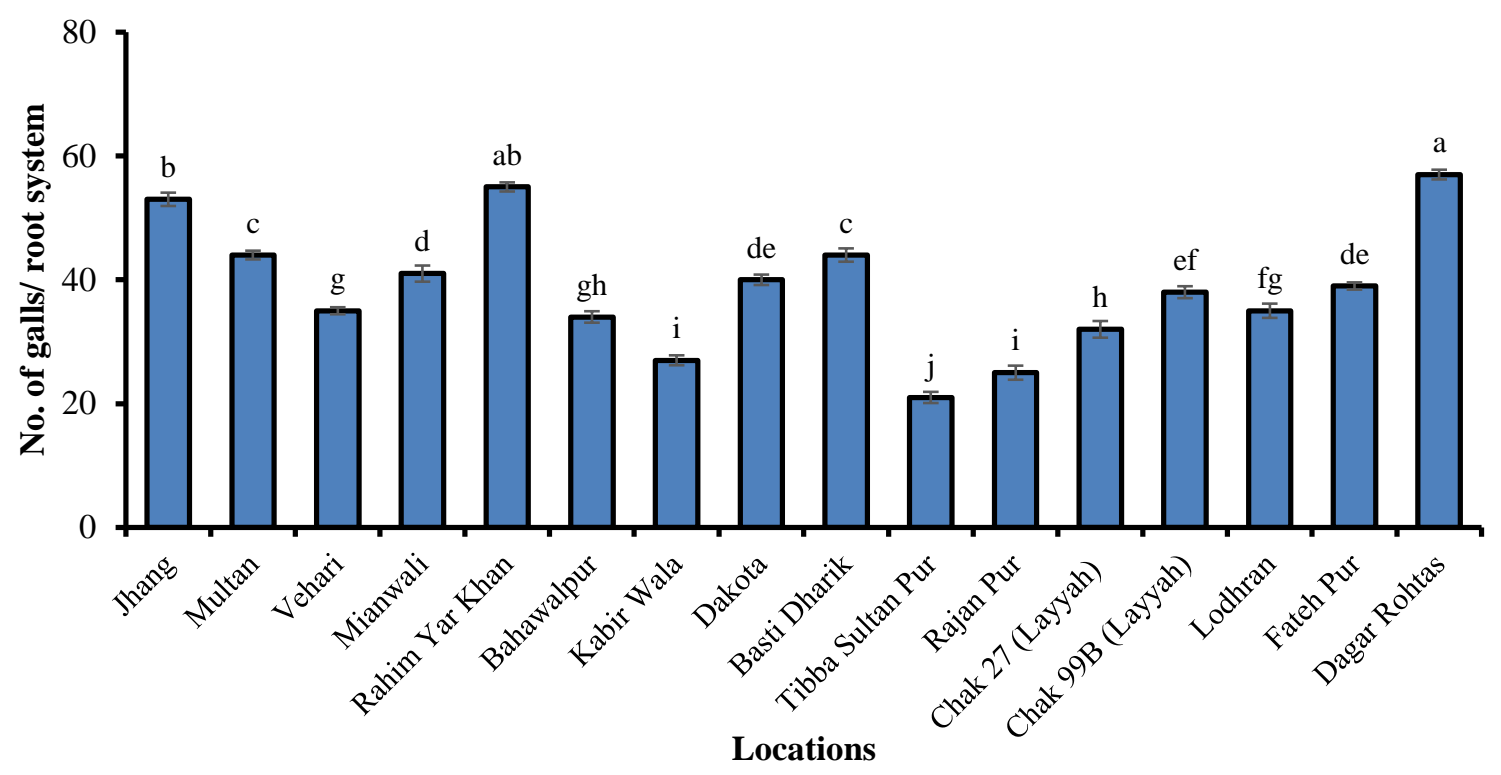

Figure 10. The number of galls counted in cotton root samples collected from different locations. Means sharing similar letters are statistically non-significant at $(\mathrm{P}<0.05)$ according to Least Significant Difference and vertical bars give standard error (SE) of means.

different from others. Mianwali (57), both Dakota and Fateh Pur $(55)$ which was non significantly $(\mathrm{P}<0.05)$ different from others. Minimum number of females in Tibba sultan (30) as shown in Fig. 9.

No of galls: The maximum number of galls was recorded in Jhang (53), both Multan and Basti Dharik (44) followed by Mianwali (41), Dakota (40), Fateh Pur (39) Layyah Chak 99b (38), Vehari and Lodhran (35), Bahawalpur (34), Layyah Chak 27 (32), Kabirwala (27), Rajan Pur (25) and Tibba sultan (21) as shown in Fig. 9. Dagar Rohtas (57) followed by Rahim yar khan (55) which was non significantly $(\mathrm{P}<0.05)$. They are parameters of all locations were significantly different from each other at $(\mathrm{P} \leq 0.05)$ as shown in Fig. 10 .

\section{DISCUSSION}

Root-knot nematodes are a major pathogen in tropical and subtropical areas of the world.

Meloidogyne incognita Chitwood, is an important and common plant parasitic nematodes of subtropical and tropical areas are also present in Pakistan (Sasser et al., 1983; Siddique et al., 2004; Anwar et al., 2007). Root-knot nematodes preferred habitat is a hot climate and sandy loam soils (Maqbool, 1987). In present studies root knot nematodes were found present in Pakistani soils as previous researchers indicated their presence in the fields of cotton and other crops. (Anwar and McKenry, 2010; Kamran et al., 2010). Prevalence of cotton wilt has recorded the maximum in areas of Vehari, Kabir Wala and Rajan Pur while the maximum incidence in both Multan and Tibba Sultan Pur. The results of the survey of different locations indicated that the maximum nematode population was recorded in Bahawalpur, followed by which Vehari, Multan, and Dagar Rohtass. The highest population of Juvenile and female was recorded in Dagar Rohtass. Processing of samples, isolations and identifications of pathogens was done. In the United States of America, seventeen states of cotton were infected with root-knot nematodes (Blasingame and Patel, 2005).

The root-knot nematode was distributed in Pakistan's soil as M. arenaria $8 \%, M$. incognita $52 \%$, M. hapla $7 \%$, M. javanica $31 \%$ and species other than Meloidogyne are 2\% (Maqbool, 1987). Root-knot nematodes can survive in diverse plant hosts which are more than 3000 plant species across the world and Pakistan (Reddy, 1983: Abad et al., 2003). Among different hosts, root-knot nematodes attack cotton roots and galls produced which leads to blockage of vascular tissues and inhibition of uptake of water and nutrients that ultimately cause yield reduction (Veech, 1984). Root knot nematodes affect cotton roots and reduce the water, nutrients uptake from soil (Kirkpatrick et al., 1991). Due to root-knot nematode infection, cotton plants growth-retarded that ultimately decrease the yield (Thomas and Kirkpatrick, 2001). Approximately about $81 \%$ share of cotton production in Punjab, Pakistan (Nia, 2000). del Prado-Vera et al., 2001 sampled 47 localities from 18 states of Mexico. A total population of 56 populations were obtained from $60.7 \%$ belong to $M$. incognita, $21.4 \%$ to $M$. arenaria, $12.5 \%$ to $M$. javinica and $53 \%$ to $M$. hapla. Root-knot nematode $(M$. incognita) alone are also potential pathogens of the cotton crop as they can break resistance in wilt resistant cotton cultivars to some extent (Carling et al., 1989) and they also impose stress and weakens the seedlings which lead to the 
death or the predisposal of seedlings (Cauquil and Shephered, 1970). Nematodes also decrease root growth.

In the present study, cotton-growing regions of Punjab was surveyed to determine the prevalence, incidence of root-knot nematodes with complex of other fungal pathogens on roots, stem, leaves and soils. Among isolates nematode, fungal complexes involve the interaction of root-knot nematodes and soil-borne fungi. Most of the infected plants were also infected with soil-borne fungi. In the light of facts obtained from works of various scientists, Fusarium wilt of cotton is now known as FOV-RKN Disease Complex (Smith, 1953; Jorgenson et al., 1978; Hyer et al., 1979; Jeffers and Roberts, 1993; Ulloa et al., 2016; Mathivathani et al., 2019). Due to nematode feeding and infection injury, secondary pathogens bacteria and fungi are facilitated to attack the plants (Powell, 1971).

$F$. oxysporum was the most prevalent and soil borne pathogen in cotton wilt isolated from sample (Halpern et al., 2018; Smith et al., 2001). Among different fungi $F$. oxysporum, Colletotrichum spp., Verticillium spp., Rhizoctonia spp. and Pythium spp. are main group of fungi causing severe losses. These fungi persist in the soil as chlamydospores and associated with roots of susceptible, resistant and non-cotton hosts as well as in seed. Among different root-knot species, Meloidogyne incognita is the most virulent that cause wilting in cotton (Martin et al., 1956). Meloidogyne incognita and different soil borne fungi $F$. oxysporum, Colletotrichum spp., Verticillium spp., Rhizoctonia spp. and Pythium spp. affect the growth and yield of cotton. Due to reproduction potential, $M$. incognita cause severe damage to roots and difficult to control. F. oxysporum, Verticillium, Colletotrichum spp. and Rhizoctonia spp were common in most of the cotton plants infected with $M$. incognita. Different diseases occur in cotton, among these, wilt of cotton caused by $F$. oxysporum, Verticillium, Colletotrichum and Pythium cause 30-50\% yield reduction. In the results of the present study, the survey confirmed that the highest frequency percentage of fungi present in roots indicated that having a higher density of nematodes which causing the wilt of cotton.

RKN-FOV is a complicated interaction (Colyer, 2007) and many models have been proposed to elaborate on the mechanism of this interaction. The early investigators believed that nematodes act as vectors for fungi thereby transmitting FOV to the conductive tissue of the cotton plants (Mai and Abawi, 1987; Colyer, 2007). Another hypothesis was about entry points that nematodes provide for fungal entry into the plant (Abo-El-Dahab, 1961; Smith, 1953; Robinson, 2008). According to this hypothesis, second juvenile stage $(\mathrm{J} 2)$ of root-knot nematodes penetrate the roots from the tips or near the tips. As juveniles approach the vascular tissues, hypertrophy (cell enlargement) and hyperplasia (increase in the number of cells) take place (Robinson, 2008). The enlarged cells give a knot like an appearance that is why this genus of nematodes is known as root-knot (Bezawada et al., 2003). Root knot nematodes are well known to induce such modifications in the host cells to facilitate the sucking of food materials through their stylet. The female inside these knots releases the egg masses. After hatching, eggs give rise to developing new $\mathbf{J} 2$ that ultimately infect the plants in their vicinity. Nematodes leave the ends of conducting vessels open, providing openings entry points for fungi (Smith, 1953; Minton and Minton, 1966). Powell (1971) observed a more pronounced synergistic effect when the fungus was inoculated 2 to 4 weeks after the inoculation of nematodes (Powell, 1971; Colyer, 2007).

Conclusion: A Survey of various cotton-growing areas revealed that wilt causing plant pathogenic fungi cause losses to vigour and damage plant. In combination with root-knot nematode, losses increase in term of vigour and yield as nematodes avenues provide. Collected information may provide a better understanding about the interaction of fungi and nematodes in cotton in Pakistan.

Acknowledgments: Authors thanks to HEC project no. 9400. The title "Relationship between fungus and nematodes associated with cotton wilt in Punjab and its integrated management".

\section{REFERENCES}

Abad, P., B. Favery, M.N. Rosso and P. Castagnone-Serena. 2003. Root knot nematode parasitism and host response: molecular basis of a sophisticated interaction. Mol. P1. Pathol. 4:217-224.

Abawi G.S. and J. Chen. 1998. Concomitant pathogen and pest interactions. In: Barker K.R., G.A. Pederson and G.L. Windham (eds). Plant and Nematode Interactions. Wisconsin, USA: American Society of Agronomy. pp.135-158.

Abo-El-Dahab and M.K. 1961.Relationship of root-knot nematodes and Fusarium-wilt of Cotton in Egyptian soils.Phytopathol. Mediterr. pp. 61-65.

Agrios, G.N. 2005. Plant Pathology, $5^{\text {th }}$ edition. Academic Press. USA. pp. 570.

Anwar, S.A. and M.V. McKenry.2010. Incidence and reproduction of Meloidogyne incognita on vegetable crop genotypes. Pak. J. Zoology. 42:135-141.

Anwar, S.A. and M.V. Mckenry. 2007. Variability in reproduction of four populations of Meloidogyne incognita on six cultivars of cotton. J. Nematol. 39:105110.

Anwar, S.A., A. Zia, M. Hussain and M. Kamran. 2007. Host suitability of selected plants to Meloidogyne incognita, in the Punjab, Pakistan. Int. J. Nematol.17:144-150.

Atkinson, G. F., 1892. Some Diseases of Cotton Alabama Polytech. Inst. Agric.-Exp. Stat. Bull. 41:61-65 
Bakhsh A, A.Q., Rao, A.A., Shahid T. Husnain, S. Riazuddin. 2009. Insect resistance and risk assessment studies in advance line of Bt. Cotton harboring Cry 1AC and Cry 2A genes. Amer. Eur. J. Agric. Environ. Sci. 6:1-11.

Bezawada, C., S. Saha, J.N. Jenkins, R.G. Creech and J.C. McCarty. 2003. SSR marker (s) associated with root knot nematode resistance gene (s) in cotton. J. Cotton Sci. 7:179-184.

Blasingame, D. and Patel, M.V. 2005. Cotton disease loss estimate committee report. 2005 Proceedings of the Beltwide Cotton Conferences. National Cotton Council of America, Cordova.

Bridge, J., S.L.J. Page and S. Jordan. 1981. An improved method for staining nematodes in roots. Roth. Exper. Report. 1:17-19.

Carling, D. E., R.W., Roncadori and R.S. Hussey. 1989. Interaction of vesicular-arbuscular mycorrhizal fungi, root-knot nematode, and phosphorus fertilization on soyabean. Plant Dis. 73:730-733.

Cauquil, J. and R. I. shepherd. 1970. Effect of root-knot nematode-fungi combinations on cotton seedling disease. Phytopathol. 60:448- 451.

Choi, Y.W., K.D. Hyde and W.H. Ho. 1999. Single spore isolation of fungi. Fungal Diver. 3:29-38.

Colyer, P.D. 2007. Current status of Fusarium wilt in the United States and future challenges. Proceedings World Cotton Research Conference 4, Lubbock, Texas, USA.pp.10-14.

Del-Prado-Vera, I. C., A. Tovar-Soto and J. A. Hernandez. 2001. Distribution of Meloidogyne species and races in Mexico. Revista Mexicana de Fitopathologia. 19:32-39.

Economic Survey of Pakistan (EPS), 2017-2018. Government of Pakistan, ministry of food, agriculture and livestock. Pakistan Bureau of Statistics, Islamabad, Pakistan.

Halpern, H.C., A.A. Bell, T.A. Wagner, J. Liu, R.L. Nichols, J. Olvey, J.E. Woodward, S. Sanogo, C.A. Jones, C.T. Chan and M.T. Brewer, 2018. First report of Fusarium wilt of cotton caused by Fusarium oxysporum f. sp. vasinfectum race 4 in Texas, USA. Plant Disease. 102:446-446.

Hartman K.M, and J.N. Sasser. 1985. Identification of Meloidogyne species on the basis of differential host test and perineal pattern morphology. In: Barker KR, Carter CC, Sasser JN, editors. Advanced Treatise on Meloidogyne. Volume II: Methodology. Raleigh, NC, USA: North Carolina State University.pp. 69-77.

Hutmacher, B., M.R., Davis, Y. Kim. 2003. Fusarium Information. University of California Cooperative Extension.pp.1-7.

Hyer, A.H., E.C. Jorgenson, R.H. Garber and S. Smith. 1979. Resistance to root-knot nematode in control of root-knot nematode-fusarium wilt disease complex in cotton. Crop Sci. 19:898-901.
Jeffers, D.P. and P.A. Roberts. 1993. Effect of planting date and host genotype on the root-knot nematode-Fusarium wilt disease complex of cotton. Phytopathology. 83:645654.

Jorgenson, E.C., A.H. Hyer, R.H. Garber and S.N. Smith. 1978. Influence of soil fumigation on the Fusarium-rootknot nematode disease complex of cotton in California. J. Nematol. 10:228.

Kamran, M., S.A. Anwar, M. Javed, S.A. Khan and G.H. Sahi, 2010. Incidence of root-knot nematodes on tomato in Sargodha, Punjab, Pakistan. Pak J. Nematol. 28:253262.

Kirkpatrick, T.L., D.M. Oosterhuis and S.D. Wullschleger. 1991. Interaction of Meloidogyne incognita and water stress in two cotton cultivars. J. Nematol. 23:462-467.

Kulkarni, S. P. 2006. Studies on Fusarium oxysporumf. sp. gladioli (Massey Snyd. and Hans.) causing wilt of gladiolus. Ph.D. Thesis submitted to the University of Agricultural Sciences, Dharwad.

Lutfunnessa, R.J.F. and S. Shamsi. 2011. Fungal diseases of cotton plant (Gossypium hirsutum L.) in Bangladesh. Dhaka Univ. J. Biol. Sci. 20:139-146.

Mai, W.F. and G.S. Abawi. 1987. Interactions among rootknot nematodes and Fusarium wilt fungi on host plants. Annu. Rev. Phytopathol. 25:317-338.

Maqbool, M.A. 1987. Classification and distribution of plant parasitic nematodes in Pakistan. Pak. J. Nematol.5:15-17.

Martin, W.J., L.D. Newson and J.E. Jones. 1956. Relationship of nematodes to the development of Fusarium wilt in cotton. Phytopathol.46:285-289.

Mathivathani, C., K. Poornima, P. Kalaiarasan and M. Muthamilan. 2019. Survey and Pathogenicity of Fusarium Wilt Disease in Cotton Fields of Tamil Nadu. Int. J. Curr. Microbiol.Appl. Sci. 8:1720-1726.

Mckerny, M.V. and P.A. Roberts. 1985. Phytonematology study guide cooperative Extension University of California. Division of Agriculture and Natural Resources.pp. 40-45.

Minton, N.A. and E.B. Minton. 1966. Effect of root knot and sting nematodes on expression of fusarium wilt of cotton in 3 soils. Phytopath.56:319.

Nia, S.M.A. (2000). Cotton: An important cash crop. Karachi, Pakistan: Pakistan \& Gulf Economist Magazine. Available on the World Wide Web: http://www.pakistaneconomist.com/issue2000/issue18/c ontents.htm.

Niu C, H.E. Lister, B. Nguyen, T.A. Wheeler and R.J. Wright. 2008. Resistance to Thielaviopsis basicola in the cultivated a genome cotton. Theor. Appl. Genet.117:1313-1323.

Powell, N.T. (1971) Interactions between nematodes and fungi in disease complexes. Ann. Rev. of Phytopathol. 9: 253-74. 
Powell, N.T. 1971. Interactions between nematodes and fungi in disease complexes. Annu. Rev. Phytopathol.9:253274.

Prasad, N. and G.W. Padwick. 1939. The genus Fusarium II. A Species of Fusarium as a cause of wilt of gram (Cicer arietinum L). Ind. J. Agri. Sci. 9:371-380.

Rao, S., S. Danish, S. Keflmariam, H. Tesfagergish, R. Tesfamariam and T. Habtemariam. 2016. Pathological survey on disease incidence and severity of major diseases on Tomato and Chilli crops grown in Sub Zoba Hamelmalo, Eritrea. Int. J. Res. agric. Sci. 2:20-31.

Reddy, P.P. 1983. Plant nematology. Agricole Publishing Academy, New Delhi, India. Pp.1-9.

Robinson, A.F. 2008. Nematode management in cotton.Integrated Management and Biocontrol of Vegetable and Grain Crops Nematodes.Springer.pp.149182.

Saxena, M. C., and K. B. Singh, 1987: The Chickpea, 399 p. C.A.B Int. Wallingford, UK.

Sial K.B., A.D. Kalhoro, M.Z. Ahsan, M.S. Mojidano, A.W. Soomro, R.Q. Hashmi, A. Keerio. 2014. Performance of different upland cotton varities under the climatic condition of central zone of Sindh. Amer.-Eur. J. Agric. Environ. Sci. 14:1447-1449.

Smith, A.L. 1953. Fusarium and nematodes on cotton. USDA Yearb. Plant Dis. 292-298.

Smith, S.N., J.E. DeVay, W.H. Hsieh and H.J. Lee, 2001. Soil-borne populations of Fusarium oxysporum F. sp. vasinfectum, a cotton wilt fungus in California fields. Mycologia. 93: 737-743

Taylor, C.E. 1990. Nematode interactions with other pathogens. Ann. App. Bio. 116:405-16.

Thomas, S.H. and T.L. Kirkpatrick. 2001. Root knot nematodes. In: Kirkpatrick T.L., C.S. Rothrock (Eds).
Compendium of cotton diseases. $2^{\text {nd }}$ edition. St. Paul, MN: APS Press. pp. 40-42.

Ulloa, M, R.B. Hutmacher, P.A., Roberts, S.D. Wright, R.L., Nichols and D.R. Michael. 2013. Inheritance and QTL mapping of Fusarium wilt race 4 resistance in cotton. Theor. Appl. Genet. 126:1405-1418.

Ulloa, M., C. Wang, S. Saha, R.B. Hutmacher, D.M. Stelly, J.N. Jenkins, J. Burke and P.A. Roberts. 2016. Analysis of root-knot nematode and fusarium wilt disease resistance in cotton (Gossypium spp.) using chromosome substitution lines from two alien species. Genetica. 144:167-179.

Veech, J.A. 1984. Cotton protection practices in the USA and world. Section C. Nematodes p.309-330 in the R. J. Kohel and C. Flewis eds. Cotton. Agronomy monograph no. 24. ASSA-SSSA Madison, WI 1990. Nematodes parasitism of cotton. Fla. Dept. Agric and consumer Serv, Div. Plant Intd. Nema. Circ. No. 183.

Wang, C., Ulloa, M., Mullens, T.R., John, Z.Y. and Roberts, P.A., 2012. QTL analysis for transgressive resistance to root-knot nematode in interspecific cotton (Gossypium spp.) progeny derived from susceptible parents. PloS one. 7:34874.

Warcup, J.H. 1955. Isolation of fungi from hyphae present in soil. Nature. 175:953-954.

Whitehead, A.G. and A.K. Hemming. 1965. Comparison of quantitative method of extracting the small vermiform nematode from soil. Ann. Appl. Biol. 55: 25-38.

Wosten, A.B., M.M. Serge, H.S. Johannes and J.H. Wessel. 1991. Localization of growth and secretion of proteins in Aspergillus niger. Microbiol. 137:2017-2023.

Zareen, A., J.M. Zaki and N. Javed. 2003. Nematicidal activity of ginger and its effect on the efficacy Pasteuria penetrans for the control of root knot nematodes. Asian J. Plant Sci. 2:858-860. 